\title{
Research on Evaluation Model of Equipment Support Logistics plans in Wartime Based on Topsis
}

\author{
Yang Fan ${ }^{1, b}$, Zhao Zhanbiao, a , Jiang Tianyuan ${ }^{2, c}$, Dong Yucai ${ }^{2, d}$ \\ ${ }^{1}$ Scientific Research Department, Academy of Armored Forces Engineering, Beijing, 100072, China \\ 2 Department of Foundation, Academy of Armored Force Engineering, Beijing 100072, China \\ a673729309@ @q.com, bJohnath @sohu.com, cjty1205@163.com, ddongyucai_001@163.com
}

Keywords: Topsis, Equipment support distribution plan in wartime, Comprehensive evaluation.

\begin{abstract}
Evaluation of equipment `support distribution 'plan in wartime scientifically and accurately is crucial to improve the efficiency of distribution and meet the demand of war timely. In this paper Topsis is adopted to conduct comprehensive evaluation of the equipment support distribution plan at war time. Topsis is an effective resolution of multiple objectives, nearly an ideal resolution plan, which effectively avoids the difficulty in combining the multiple evaluation parameters into a single efficacy value and provides a new way to evaluate the equipment support distribution plan in wartime.
\end{abstract}

\section{Introduction}

As the future warfare becomes more informationized, the ever-changing battlefield has higher requirements of the equipment support in wartime [1]. On the battlefield under pressing time and heavy tasks, scientific equipment support distribution plan, good use of resources available and accurate rational equipment and materials support at the earliest possible time make great difference in the war [2-3]. Literature [4] have combined the logistic distribution routes and the heritage simulate annealing, taking into comprehensive consideration the evaluation of multiple objectives of the logistic distribution routes in wartime. Literature [5] have built distribution route arrangement model for multiple distribution centers with the capacity of random delays and losses in wartime, offering the ant colony algorithm based on the random model to optimize the distribution routes. Based on the characteristics of the equipment support logistic distribution in wartime, Literature [6] have set up the evaluation index system and on the basis of the analysis of grey relational grade, they have built the evaluation model for equipment support logistic plan by using the comentropy theory to assign values to the indices. However, the theoretical foundation for the grey relational grade quantitative model is relatively narrow, so it is improper to determine the relativity among the factors purely from the angle of relative curve shapes. At the same time, the relativity obtained from the analysis of the grey relativity is always positive, which cannot reflect the relations among things in an overall way.

\section{Topsis}

Topsis, abbreviated for "technique for order preference by similarity to ideal solution", was first brought forth by Hwang and Yoon in 1980[7]. Then its concept was used to deal with multiple objective decisions in 1994 by Laietal, thus developing into a commonly used method in the multiple-objective decision analysis in system engineering which is adopted in many fields such as performance evaluation, decision-making and management[8-10]. It can be used to conduct efficiency evaluation of things with multiple properties and of different kinds [11-14]. This paper then adopts Topsis to carry out comprehensive evaluation of the equipment support logistic distribution plan in wartime. 


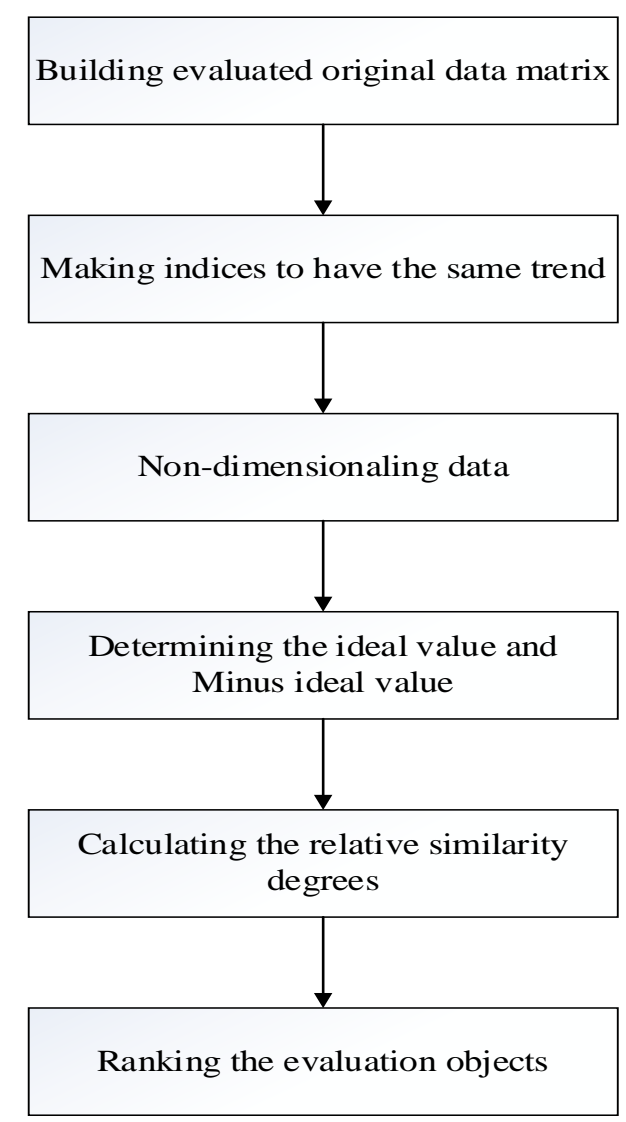

Figure 1 the step of Topsis method

Topsis [15] works in this way as the figure 1 above: firstly, it finds out the optimal and worst plans (which are represented by the optimal and worst vectors respectively) in the limited plans on the basis of original data matrix after normalization; secondly, it calculates the distances of the evaluation objects from the optimal and worst plans; thirdly, it obtains the relative similarity, which is used as the standard to judge their excellence.

\section{Data structure}

Suppose there are $\mathrm{n}$ objects or plans to be evaluated, each with $\mathrm{m}$ evaluation indices. The original data are shown in the following matrix:

$$
X=\left[\begin{array}{cccc}
x_{11} & x_{12} & \cdots & x_{1 m} \\
x_{21} & x_{22} & \cdots & x_{2 m} \\
\vdots & \vdots & \vdots & \vdots \\
x_{n 1} & x_{n 2} & \cdots & x_{n m}
\end{array}\right]_{n^{*} m} .
$$

\section{Making the indices to have the same trend}

There are positive and inverse evaluation indices. The former includes the indices of efficiency and the latter the ones of costs. In evaluation, the indices are required to have the same trend, which is done by converting the inverse indices into positive ones as a common practice. In conversion, the method of reciprocal (that is, $1 / x$ ) is most used for absolute number indices while the method of difference (that is, $1-x$ ) for relative number indices. The data matrix that has been converted is still marked as $\mathrm{X}[16]$.

Non-dimensionaling data

The data matrix after non-dimensionalization is marked as $\mathrm{Y}$, 
$Y=\left[\begin{array}{cccc}y_{11} & y_{12} & \cdots & y_{1 m} \\ y_{21} & y_{22} & \cdots & y_{2 m} \\ \vdots & \vdots & \vdots & \vdots \\ y_{n 1} & y_{n 2} & \cdots & y_{n m}\end{array}\right]_{n \times m}$

in which

$$
z_{i j}=\frac{x_{i j}}{\sqrt{\sum_{i=2}^{n} x_{i j}^{2}}} \quad(i=1,2, \cdots, n ; j=1,2, \cdots, m) .
$$

\section{Determining the ideal value and minus ideal value}

Determining the ideal value and minus ideal value of the indices, which form ideal value vector $Z^{+}$and minus ideal value vector $Z^{-}$,

$$
\begin{aligned}
& Z^{+}=\left(z_{1}^{+}, z_{2}^{+}, \cdots, z_{m}^{+}\right), \\
& Z^{-}=\left(z_{1}^{-}, z_{2}^{-}, \cdots, z_{m}^{-}\right) .
\end{aligned}
$$

in which

$$
\begin{aligned}
& z_{j}^{+}=\max \left(z_{1 j}, z_{2 j}, \cdots, z_{n j}\right), \\
& z_{j}^{-}=\min \left(z_{1 j}, z_{2 j}, \cdots, z_{n j}\right) \quad(j=1,2, \cdots, m) .
\end{aligned}
$$

\section{Calculating the relative similarity degrees}

Calculating the relative similarity degrees between the index value vector and the ideal value vector of the evaluation objects with Euclidean distance formula, that is,

$$
\begin{aligned}
& D_{j}^{+}=\sqrt{\sum_{j=1}^{m}\left(z_{i j}-z_{j}^{+}\right)^{2}} \quad(i=1,2, \cdots, n) . \\
& D_{j}^{-}=\sqrt{\sum_{j=1}^{m}\left(z_{i j}-z_{j}^{+}\right)^{2}}
\end{aligned}
$$

Calculating the relative similarity degrees between the index value vector and the ideal value vector of the evaluation objects [17]:

$$
C_{i}=\frac{D_{i}^{-}}{D_{i}^{+}+D_{i}^{-}} \quad\left(0 \leq C_{i} \leq 1 ; i=1,2, \cdots, n\right) .
$$

\section{Ranking the evaluation objects}

Ranking the evaluation objects in the order of excellence based on the degrees of similarity.

Since $0 \leq C_{i} \leq 1$, when the index vector of the evaluation object becomes the ideal value vector, $C_{i}=1$; when the index vector of the evaluation object becomes the minus ideal value vector, $C_{i}=0$ 。 The closer $C_{i}$ is to 1 , the further forward the corresponding evaluation object shall be put in the ranking.

\section{Evaluation of the equipment support distribution plan in wartime}

Literature [6] points out evaluation indices shall be set rationally in the index system for logistic plan when evaluating various equipment support logistic plans in wartime comprehensively and scientifically. Therefore, after combined with needs and features of wartime equipment support, the final evaluation indices shall include time spent (K1), concealment in distribution (K2), influences by the weather (K3), anti-strike capacity (K4) and cost (K5), showed as the figure 2. 


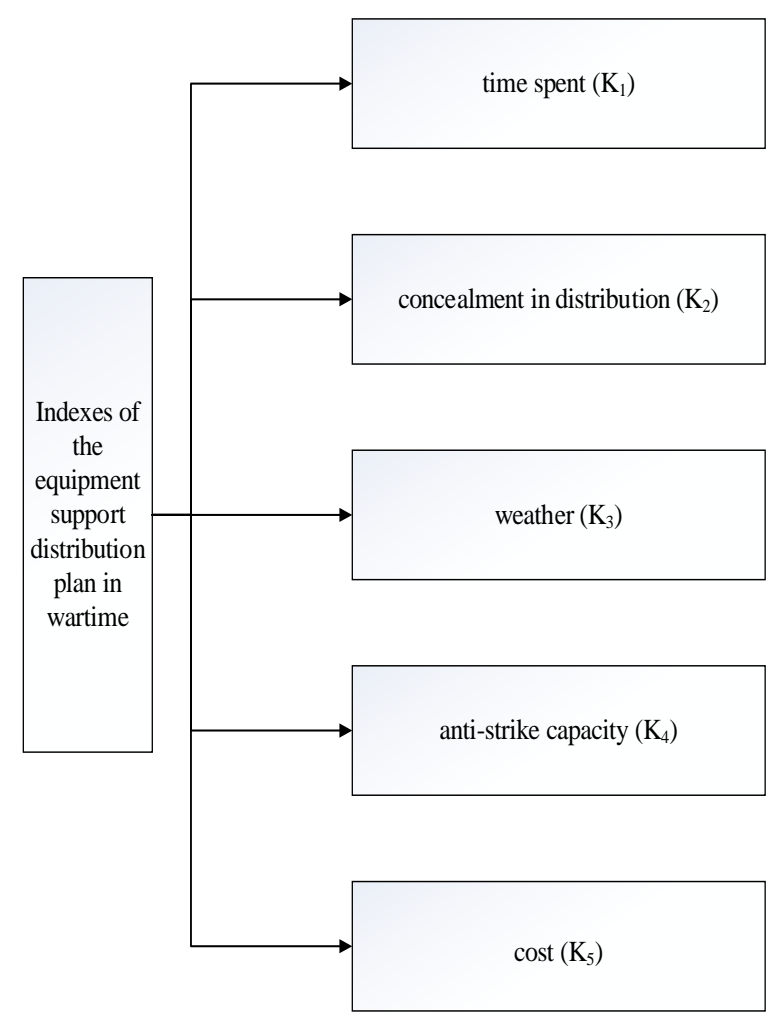

Figure 2 indexes of the equipment support distribution on plan in wartime

Then Literature [6] provides the basic index parameters of the equipment support distribution plan in wartime with 3 cases of distribution plans as shown in Table 1.

Table 1 Index parameters of the equipment support distribution plan in wartime

\begin{tabular}{cccccc}
\hline & $\mathrm{K}_{1}$ & $\mathrm{~K}_{2}$ & $\mathrm{~K}_{3}$ & $\mathrm{~K}_{4}$ & $\mathrm{~K}_{5}$ \\
\hline $\mathrm{a}$ & 86.7 & 83 & 93.3 & 93.3 & 88.3 \\
$\mathrm{~b}$ & 85.7 & 85.7 & 90 & 95.3 & 88.7 \\
$\mathrm{c}$ & 83.3 & 83.3 & 88.3 & 91.7 & 89 \\
\hline
\end{tabular}

The original matrix is as follows

$$
X=\left[\begin{array}{ccccc}
86.7 & 83 & 93.3 & 93.3 & 88.3 \\
85.7 & 85.7 & 90 & 95.3 & 88.7 \\
83.3 & 83.3 & 88.3 & 91.7 & 89
\end{array}\right] .
$$

Non-dimensionalize the data to form the following weighted matrix:

$Z=\left[\begin{array}{lllll}0.5872 & 0.5704 & 0.5948 & 0.5765 & 0.5750 \\ 0.5804 & 0.5890 & 0.5738 & 0.5888 & 0.5776 \\ 0.5642 & 0.5725 & 0.5630 & 0.5666 & 0.5795\end{array}\right]$.

Determine the index ideal value and minus ideal value:

So the ideal value is

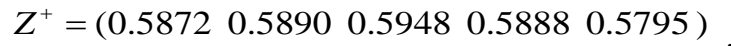

and the minus ideal value is

$Z^{+}=\left(\begin{array}{lllll}0.5642 & 0.5704 & 0.5630 & 0.5666 & 0.5750\end{array}\right)$.

As for the distance and the relative similarity, refer to Table 2. 
Table 2 Ranking of the equipment support distribution plans in wartime based on degrees of excellence

\begin{tabular}{ccccc}
\hline Plan & $\mathrm{D}_{+}$ & $\mathrm{D}_{-}$ & Statistics $\mathrm{C}_{\mathrm{i}}$ & Ranking \\
\hline $\mathrm{a}$ & 0.0228 & 0.0405 & 0.6405 & 1 \\
$\mathrm{~b}$ & 0.0222 & 0.0350 & 0.6123 & 2 \\
$\mathrm{c}$ & 0.0481 & 0.0050 & 0.0942 & 3 \\
\hline
\end{tabular}

It can be seen from Table 2 that the ranking of the equipment support distribution plans in wartime is as follows:

$a>b>c$.

The above ranking is absolutely the same as what is shown in Literature [3], which demonstrates the rationality of Topsis. According to the above ranking, Plan a gets the best comprehensive evaluation result, Plan c the worst one while Plan b is between them.

\section{Summary}

Topsis is a commonly adopted method for decision making in the multiple-objective decision analysis in system engineering. It uses the original data more fully with only small loss of information and reflects the excellence degrees of different properties quantitatively, thus featured as vivid, simple and reliable, which avoids the subjectivity of determining index weights with traditional scoring methods, such as experts scoring. Therefore, Topsis helps get a result more scientific and objective, thus providing a new way to conduct comprehensive evaluation of the equipment support distribution plans in wartime.

\section{References}

[1] XU cheng, HUANG Jin-yuan, DU Xiao-ming, YANG Jian, A Research on Reliability Evaluation of Wartime Equipment Supply Chain [J]. Logistics Sci-Tech, 2009(7), p.118-120.

[2] SUN Bao-chen, JIA Xi-sheng, CHENG Zhong-hua, WANG Ya-bin, Study on Equipment Support Process Modeling and Simulation in Wartime [J]. Command Control \& Simulation, 2012(2), p.97-104.

[3] ZU Zheng-hu, ZHOU Jing-lun, SUN Quan, Sustaining Support Capability Evaluation of Weapon Equipment in Wartime [J]. Computer Simulation, 2008 (3), p.6-8.

[4] LIU Li-bo, XU Guo-yin, YUAN Jing. Researches on Optimization of Logistics Distributing Routing Routes in Wartime Based on Genetic Simulated Annealing Algorithm [J]. Railway Transport and Economy, 2007, (7), p.71-74.

[5] Li Wang, DAI Ming-qiang. Optimization of Multiple Depots Vehicle Routing Problem with Stochastic Loss and Delay [J]. Fire Control \& Command Control, 2012, (2), p.184-189.

[6] LV Li-bo, ZHANG Shuai, Evaluation Methods of Equipment Support Logistics Solutions in Wartime [J]. Journal of Sichuan Ordnance, 2010, (10), p.51-52.

[7] Yoon k, The propagation of error in multiple - attribute decision analysis: a practical approach [J], Journal of the operational Research Society, 1989, 40(7), p.681-686.

[8] LV Gui-tian, WU Ci-fang, Li Guan, CHEN Mei-qiu, Coordination Evaluation of Urban Developmrnt and Water Environment in Gan Jiang Based on Topsis [J]. Resources \& Industries, 2014, 16(4), p.83-89.

[9] YAO Zhi-gang, WANG Yuan-qing, TIAN Gang, Ranking Perceptions of Taxi Service Quality of Participants with Topsis [J]. Journal of Wuhan University of Technology (Transportatio Science \& Engineering), 2014(1), p.85-88. 
[10] XU Da, LI Chuang, LI Yang, WANG Bao-qi, Research on the Comprehensive Evaluation of Equipment Maintainability Qualitative Indexes Based on Topsis [J]. Aerospace Control,2014,(5).

[11] LIU Chong-hong, Topsis Method in the Application of Atmospheric Environmental Quality Assessment [J], Arid Environmental Monitoring, 1993, 7(4), p.230-232.

[12] MA Li-ping, Groups Decision Method Based on TOPSIS Method[J], Journal of LIaoNing Institute of Technology, 1999,19 (2) , p.71-74

[13] HUA Dang-ling, Topsis Method of Multi-Aims Decision-making and Application [J], Computer and Agriculture, 1999(4), p.27-28.

[14] QIAN Shu-guang, Decision-making Research on Choosing Military Support Establishment Location Based on Improved TOPSIS Method [J], Ship Electronic Engineering, 2008(8), p.34-36.

[15] TANG Guang-hua, Estimate and Extend of Topsis Method [J], Zhe Jiang Statistics, 1988(2), p.12-14.

[16] XIAO Yun-mei, Research on the optimization of road freight hub layout based on Topsis [J]. Technology \& Economy in Areas of Communications, 2013 (6), p.66-69.

[17] DING Bo-qun, ZHANG Xuan, SHI Hui-min, Evaluation on Bus Line Layout Projects Basedon TOPSIS and Its Software Implementation-—A Planned Bus Line as an Example in Harbin[J]. Forest Engineering, 2013(6), p.123-128. 